\title{
Soluções de alho (Allium sativum L.) no controle de nematódeos gastrintestinais em bovinos jovens da raça Holandesa
}

\author{
PARRA, C.L.C. ${ }^{*} ;$ OLIVO, C.J.'; AGNOLIN, C.A.'; SANGIONI, L.A.2; BUZATTI, A.2; PIVOTO, F.L.2; \\ 'Universidade Federal de Santa Maria, Centro de Ciências Rurais, Departamento de Zootecnia, Santa Maria, \\ RS, Brasil, CEP 97105-900; *liedaparra@ymail.com 2Universidade Federal de Santa Maria, Departamento de \\ Medicina Veterinária Preventiva, Laboratório de Doenças Parasitárias Santa Maria, RS, Brasil.
}

\begin{abstract}
RESUMO: Para avaliar o efeito da atividade anti-helmíntica do alho suplementado, foram utilizadas 24 bezerras e novilhas da raça Holandesa, naturalmente infectadas. As soluções foram preparadas triturando-se o alho, $(50 \%)$, mais água ou álcool $92^{\circ},(50 \%)$, administrandose oralmente aos animais. Os tratamentos $(\mathrm{T})$ foram constituídos pelo grupo controle negativo (T1); extrato alcoólico de alho a $60 \mathrm{~g}$ e $120 \mathrm{~g} / 100 \mathrm{Kg}$ de peso vivo (T2) e (T3); extrato aquoso de alho a 60 e $120 \mathrm{~g} / 100 \mathrm{~kg}$ de peso vivo (T4) e (T5); e o grupo controle positivo com albendazol a $10 \%$ (T6). Os tratamentos fitoterápicos foram repetidos a cada 14 dias, caso a infecção fosse superior a 400 ovos por grama de fezes (OPG). A técnica de coprocultura quantitativa e qualitativa foi empregada para avaliar a eficácia anti-helmíntica dos tratamentos. Foram observadas diferenças entre os grupos controle e tratados para OPG e na porcentagem relativa de larvas infectantes e desenvolvimento larval. $\mathrm{O}$ uso das soluções de alho demonstrou controle parcial de nematódeos gastrintestinais.
\end{abstract}

Palavras chave: Allium sativum, gado leiteiro, fitoterapia, helmintos.

ABSTRACT: Garlic (Allium sativum L.) Solutions In Control Of Gastrointestinal Nematodes In Young Holstein Cattle. Twenty four Holstein calves and heifers naturally infected were used to evaluate the effect of garlic supply on helminth control. The solutions consisted to triturated garlic, $(50 \%)$, plus water or alcohol $92^{\circ},(50 \%)$, orally-administrable on the animals. Treatments (T) consisted of a negative control (T1), garlic at 60 (T2) and $120 \mathrm{~g}$ on alcoholic extract (T3); 60 (T4) and $120 \mathrm{~g}$ on aqueous extract/100kg of life weight (T5) and the positive control with albendazol at $10 \%$ (T6). The phytotherapic treatments were repeated after $14^{\text {th }}$ day if the infection was superior to 400 eggs per gram of faeces (EPG). The quantitative and qualitative coproculture technique was performed to evaluate the anthelmintic efficacy of the treatments. Differences between treated and control groups were observed in EPG and in relative percentage of infective larvae and the larval development. Partial control was found with the garlic solutions on gastrointestinal nematodes.

Key words: Allium sativum, dairy cattle, helminths, phytotherapy.

\section{INTRODUÇÃO}

As doenças parasitárias contribuem significativamente para a redução da eficiência produtiva dos animais (Bianchin e Catto, 2004), implicando em grandes perdas econômicas (Grisi et al., 2002; Cardoso et al., 2012), com destaque para as helmintoses. As infecções por nematódeos gastrintestinais causam prejuízos extremamente significativos à criação de ruminantes, sendo que o déficit produtivo causado por infecções sub-clínicas acarreta grande impacto econômico (Forbes et al., 2002), limitando a produção de leite, reduzindo o ganho de peso e a conversão alimentar; além de comprometer o desempenho reprodutivo e o sistema imunológico (Costa et al., 2004).

Para o controle convencional dessas parasitoses, geralmente, são usados produtos químicos. No entanto, também são observados efeitos adversos do uso desses medicamentos, podendo acarretar malefícios ao hospedeiro, ao homem, que consome os produtos de origem animal, e ao ambiente (Chagas et al, 2003). Acrescentase, ainda, problemas com o desenvolvimento da resistência dos parasitas aos quimioterápicos utilizados (Furlong et al., 2004), notadamente pelo

Recebido para publicação em 15/06/2011

Aceito para publicação em 27/03/2014 
uso indiscriminado e inadequado desses produtos (Sequeira, 2001), pois pequenas diferenças nas formulações podem eventualmente causar importantes e significativas alterações na atuação e consequente eficácia dos helminticidas (Borges et al., 2003). Nesse contexto, há grande dificuldade em se conviver com a verminose em sistemas de produção convencional. Considerando o sistema orgânico ou agroecológico, o controle parasitário é um grande desafio (Cabaret, 2003). Nestes tipos de agricultura, o uso de quimiossintéticos artificiais é estritamente limitado, podendo ser usados quando indispensáveis, após diagnóstico de um grau alto de severidade à saúde do animal, sendo que após o tratamento o período de carência a ser respeitado, para que os produtos e subprodutos dos animais tratados possam voltar a ter o reconhecimento como orgânicos, deverá ser duas vezes o período de carência estipulado na bula do produto.

Nesse contexto, a fitoterapia é considerada uma alternativa importante no controle de parasitas (Catto et al., 2009), podendo reduzir os impactos econômicos e ambientais verificados com uso dos produtos convencionais (Avancini, 1994). Agrega-se também a isso que há um aumento da produção de alimentos orgânicos no Brasil e no mundo (Santos et al., 2012). Ressalta-se ainda que a utilização de fitoterápicos em sistemas convencionais de produção, como parte da estratégia de controle de parasitas, pode elevar a vida útil dos produtos químicos (Vieira e Cavalcante, 1999) e, pelo fato dos fitoterápicos possuírem associações de compostos de vários princípios ativos, pode tornar a resistência um processo mais lento (Roel, 2002).

Dentre os fitoterápicos para o controle de parasitas, destaca-se o alho (Amaral et al., 2006). No entanto, mesmo havendo referências seculares de seu uso terapêutico em animais domésticos, as pesquisas são escassas, apresentando grandes diferenças na constituição de soluções e forma de uso, havendo grande variabilidade de metodologias e de resultados. Assim, o objetivo desta pesquisa foi avaliar o efeito de distintas soluções de alho, a partir de extração alcoólica e aquosa, administradas oralmente no controle de nematódeos gastrintestinais em bovinos.

\section{MATERIAL E MÉTODOS}

A pesquisa foi realizada entre os meses de abril e dezembro de 2009, nos laboratórios de Bovinocultura de Leite (Departamento de Zootecnia - DZ) e de Doenças Parasitárias (Departamento de Medicina Veterinária Preventiva), pertencentes à Universidade Federal de Santa Maria - UFSM (RS), situada na região da Depressão Central, com clima subtropical úmido com médias de temperatura diária de $17^{\circ} \mathrm{C}$ e precipitação mensal de $112 \mathrm{~mm}$.

Os tratamentos foram constituídos pelo grupo controle negativo (testemunha-T1), no qual os animais não receberam nenhum produto, sendo submetidos às mesmas condições de manejo e alimentação dos demais grupos: extrato alcoólico de alho a $60 \mathrm{~g}$ e $120 \mathrm{~g} / 100 \mathrm{Kg}$ de peso vivo (T2) e (T3); extrato aquoso de alho a 60 e $120 \mathrm{~g} / 100 \mathrm{~kg}$ de peso vivo (T4) e (T5); e o grupo controle positivo com albendazol a 10\% (T6). Para a preparação das soluções com alho, foram triturados bulbilhos inteiros em liquidificador com álcool etílico (92\%) à semelhança da metodologia utilizada por Vendramim e Castiglioni (2000), usando-se relação de 1:1 (Fujisawa et al., 2008), \% p/v, deixando-se a mistura em repouso por 24 horas. Após esse período, o material foi coado, usando-se peneira de malha de $1 \mathrm{~mm}$, e administrado aos animais, por via oral com seringa graduada, por beberagem. Procedimento similar foi usado nas soluções aquosas, triturandose os bulbilhos inteiros em liquidificador com água.

Para avaliação foram utilizadas 24 terneiras e novilhas da raça Holandesa, com idade entre dois e dez meses, naturalmente infectadas, pertencentes ao rebanho leiteiro do Laboratório de Bovinocultura de Leite (DZ-UFSM). Historicamente, nos últimos quatro anos, os animais do rebanho vêm sendo monitorados com avalições feitas a cada três meses, sendo que apenas os mais jovens, cerca de $30 \%$, necessitam de controle parasitário. A base da alimentação dos animais experimentais foi constituída de pastagens de ciclo hibernal, especialmente azevém (Lolium multiflorum Lam.) e estival, com pastagens do gênero Cynodon spp. A complementação alimentar diária com concentrado foi em média de $0,8 \%$ do peso vivo.

Foram feitas coletas de fezes no período pré-tratamento (-3, $-2,-1$ dia), formando-se um valor médio e após a administração dos produtos (1, 3, 7 e 11. dia). Realizaram-se contagens de ovos de nematódeos por grama de fezes (OPG) (individualmente para cada amostra fecal), pelo método de Gordon e Withlock (1939), modificado, com sensibilidade de detecção de 50 OPG; e cultivos de larvas (uma por tratamento, antes da administração dos produtos e no $7 \circ$ dia após a aplicação dos mesmos), pelo método proposto por Roberts e O'Sullivan (1950), identificando-se até 100 larvas infectantes por coprocultura (Ueno e Gonçalves, 1998). Foram selecionados animais com OPG inicial $\geq 400$, os quais foram divididos em grupos com médias de OPG pré-tratamento equivalentes, por meio de randomização estratificada. Nos grupos submetidos ao tratamento com fitoterápico, repetiuse a administração dos extratos no $14^{\circ}, 2^{\circ}{ }^{\circ}$ e $42^{\circ}$ dia, somente para aqueles que apresentavam infecção de $O P G \geq 400$. Os animais que apresentaram 
valor inferior a 400 OPG, apesar de não terem recebido outra administração, continuaram sendo monitorados como os demais dos seus respectivos grupos. No tratamento com o produto químico utilizou-se albendazol a 10\%, em dose única.

Para avaliar o comportamento dos produtos, os dados de OPG foram submetidos à análise de regressão, verificando-se o modelo mais representativo. Para a análise da eficácia, foram utilizados os dados do 7० dia após a aplicação dos produtos, valendo-se da seguinte fórmula: Eficácia $=[$ (média de OPG dos animais controle - média de OPG dos animais tratados) / média de OPG dos animais controle *100]. E, para coprocultura fez-se a comparação entre os valores de pré e pós administração dos produtos, com transformação porcentual dos dados.

O desenho experimental utilizado foi o inteiramente casualizado, com seis tratamentos, e quatro repetições (animais). Para diminuir a variabilidade dos valores, os dados foram submetidos à transformação logarítmica de base dez. Os dados foram submetidos à análise de variância e as médias comparadas entre si pelo Teste de Tukey ao nível de $5 \%$ de probabilidade do erro com auxílio do programa estatístico (SAS, 2000). Foi utilizado o seguinte modelo matemático: $Y_{i j}=\mu+T i+R j(T i)+\varepsilon i j$, em que, Yij representa a variável dependente; $i$, o índice de tratamentos; $j$, o índice de repetições; $\mu$ é a média de todas as observações; Ti é o efeito dos tratamentos; $R j(T i)$ é o efeito de repetição dentro dos tratamentos; sij é o efeito residual.

\section{RESULTADOS E DISCUSSÃO}

Avaliando-se os resultados de OPG dos quatro grupos tratados com alho (16 animais), foi

$$
\begin{aligned}
& =60 \mathrm{~g} \text { alho extração alcólica } y=-30,302+39,211 x-4,9882 x^{2} ; R^{2}=0,8996 \\
& -120 \mathrm{~g} \text { alho extração alcólica } y=-43,068+56,067 x-7,8799 x^{2} ; R^{2}=0,8753 \\
& +60 \mathrm{~g} \text { alho em extrato aquaso } y=-22,846+28,67 x-3,1315 x^{2} ; R^{2}=0,8898 \\
& -120 \mathrm{~g} \text { alho em extrato aquoso } y=-30,302+39,211 x-4,9882 x^{2} ; R^{2}=0,8996 \\
& \ldots . \text { Albendasol } y=-62,694+79,989 x-10,775 x^{2} ; R^{2}=0,9053 \\
& \ldots \text { Controle } y=-13,249+17,258 x-2,6228 x^{2} ; R^{2}=0,8631
\end{aligned}
$$

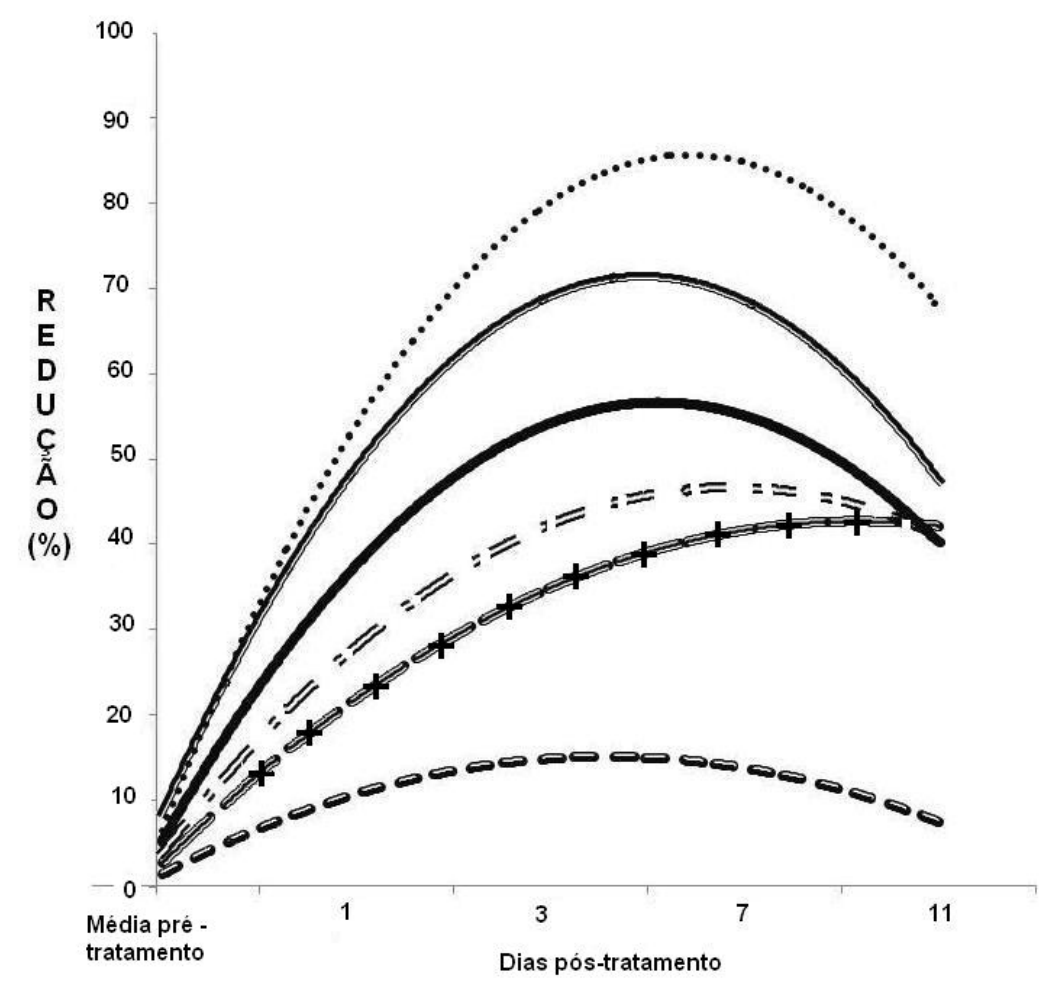

Figura 1. Percentual de redução de nematódeos, com base na média de OPG, no pré e pós-tratamento $\left(1^{\circ}\right.$, $3^{\circ}, 7^{\circ}$ e $11^{\circ} \mathrm{dia}$ ) em terneiras e novilhas de raça Holandesa, naturalmente infectadas e submetidas a distintos tratamentos com alho (Allium sativum L.) (média de 4 aplicações). Santa Maria, RS - 2009. 
necessário repetir a administração do extrato em 12,11 e 7 animais, respectivamente no $14^{\circ}, 28^{\circ} \mathrm{e}$ 42 dia, apontando comportamento diferente das soluções quanto à redução de nematódeos das terneiras e novilhas.

Considerando-se os dados médios das quatro administrações, houve um comportamento similar entre os grupos tratados com diferentes níveis de alho (Figura 1), verificando-se modelos quadráticos com início ascendente. Os dados indicam que há efeito de alho a partir do $1{ }^{\circ}$ dia após a aplicação dos produtos, havendo predominância de sua ação entre o $3^{\circ}$ e $7^{\circ}$ dia.

Com relação à eficácia dos produtos em que se usaram os dados do $7 \circ$ dia pós-tratamento, transformados em valores porcentuais (Tabela 1), observa-se que houve diferença $(P \leq 0,05)$ entre os tratamentos. Os grupos constituídos por fitoterápicos que tiveram melhor eficácia na redução de OPG foram aqueles tratados com extrato de $120 \mathrm{~g}$ de alho, havendo similaridade entre eles e o produto químico. Comparando-se, no entanto, somente os tratamentos constituídos pelos extratos, não há diferença entre eles. O produto a base de albendazol apresentou $100 \%$ de eficácia a partir do $21^{\circ}$ dia, havendo reinfecção dos animais a partir de $49^{\circ}$ dia.

Considerando-se os níveis mais elevados de alho, embora a similaridade verificada, observase melhor comportamento do nível com $120 \mathrm{~g}$ de alho sob extração aquosa pela maior facilidade de aplicação desta solução devido à menor rejeição dos animais se comparada com o extrato alcoólico. Outro motivo pelo qual se recomenda essa formulação é a elevada estabilidade da alicina em meio aquoso. Em estudo conduzido com alho macerado (50\%) em mistura $(50 \%)$ com diferentes extratores constatou-se que a estabilidade da alicina (meiavida química) em meio aquoso foi de 6,5 dias; em meio oleoso foi de 3,1 dias; e de 12; 11,9; 6,6 e 3,2 dias para a extração com álcool a 20, 50, 70 e 100\%, respectivamente (Fujisawa et al., 2008).

Os resultados verificados com as diferentes soluções de alho (Tabela 1), em média entre 41 e $61 \%$, indicam que houve ação anti-helmíntica parcial desse produto. Dentro dessa faixa reportase o trabalho conduzido por Bianchin et al. (1999), que avaliaram a eficiência do alho desidratado adicionado à mistura mineral na concentração de $2 \%$ (aproximadamente $8 \mathrm{mg} / \mathrm{kg}$ peso vivo/ dia) no controle de carrapato, mosca-dos-chifres e nematódeos, em que se observou a redução de OPG de 47,3\%. Também Batatinha et al. (2004), ao tratarem os animais com alho por um período contínuo, verificaram controle parcial de nematódeos gastrintestinais em caprinos. Por outra parte, em várias pesquisas a ação foi muito baixa ou inexistente como na experimentação feita por Fernandes et al., (2004) em que não se constatou atividade anti-helmíntica em aves usando suco de alho fresco a $10 \%$ no concentrado, correspondendo a $2 \mathrm{~g} / \mathrm{kg} / \mathrm{dia}$, por três dias consecutivos. Embora os resultados obtidos, deve-se considerar que a redução da contagem de OPG, pode acabar sendo subestimada, devido à supressão, provocada pelo produto, na excreção de ovos pelos nematódeos gastrintestinais, sem que haja, porém, a equivalente redução da carga parasitária (Condi et al., 2009).

Avaliando-se os diferentes grupos constituídos antes da aplicação dos tratamentos (Tabela 2), observa-se que há grande variabilidade na participação dos diferentes gêneros, que, em parte, pode estar associada à variação das

Tabela 1. Eficácia média de controle de nematódeos do trato gastrointestinal, com base na OPG (ovos por grama de fezes), obtido no $7 \circ$ dia pós-tratamento em terneiras e novilhas da raça Holandesa, naturalmente infectados e submetidos a distintos tratamentos. Santa Maria, RS- 2009.

\begin{tabular}{|c|c|c|c|c|c|}
\hline \multirow{3}{*}{ Grupos } & \multicolumn{5}{|c|}{ Eficácia \% } \\
\hline & \multicolumn{4}{|c|}{ Períodos de avaliação } & \multirow[t]{2}{*}{ Média } \\
\hline & 1。 & 2 & 3o & 4 4。 & \\
\hline Alho $60 \mathrm{~g}$ em álcool $/ 100 \mathrm{~kg}$ de PV & 23 & 42 & 36 & 70 & $43^{b}$ \\
\hline Alho $120 \mathrm{~g}$ em álcool $/ 100 \mathrm{~kg}$ de PV & 41 & 44 & 53 & 66 & $50^{\mathrm{ab}}$ \\
\hline Alho $60 \mathrm{~g}$ em água $/ 100 \mathrm{~kg}$ de PV & 41 & 22 & 55 & 50 & $41^{\mathrm{b}}$ \\
\hline Alho $120 \mathrm{~g}$ em água /100kg de PV & 38 & 73 & 58 & 77 & $61^{\mathrm{ab}}$ \\
\hline Albendazol & 73 & 100 & 100 & 56 & $82^{\mathrm{a}}$ \\
\hline CV (\%) & 24 & 14 & 12 & 17 & 17 \\
\hline
\end{tabular}

Os períodos de avaliação correspondem à eficácia obtida sete dias após a administração dos extratos, correspondendo ao $7^{\circ}, 21^{\circ}, 35^{\circ} \mathrm{e}$ $49 \circ$ dia da experimentação; para o albendazol, usado em dose única, fez-se o mesmo procedimento, mantendo-se as mesmas datas de coleta de fezes. a $=$ = médias seguidas por letras distintas, na coluna, indicam diferença significativa a $(P \leq 0,05)$ pelo teste de Tukey; $P V=$ Peso Vivo; CV= coeficiente de variação. 
Tabela 2. Porcentual dos gêneros de nematódeos gastrintestinais - Cooperia spp., Haemonchus spp., Trichostrongylus spp., Ostertagia spp., Oesophagostomum spp. - recuperados de culturas de larvas em fezes de terneiras e novilhas da raça Holandesa submetidas a distintos tratamentos, em 56 dias de avalição. Santa Maria, RS- 2009.

\begin{tabular}{|c|c|c|c|c|c|c|c|c|c|c|}
\hline \multirow[b]{2}{*}{$1^{\text {a }}$ Administração } & \multicolumn{2}{|c|}{ Coop. } & \multicolumn{2}{|c|}{ Haem. } & \multicolumn{2}{|c|}{ Trich. } & \multicolumn{2}{|c|}{ Ostert. } & \multicolumn{2}{|c|}{ Oesoph. } \\
\hline & Pré & Pós & Pré & Pós & Pré & Pós & Pré & Pós & Pré & Pós \\
\hline Alho $60 \mathrm{~g}$ em álcool $/ 100 \mathrm{~kg}$ de PV & 11 & 8 & 73 & 61 & 6 & 8 & 9 & 15 & 0 & 8 \\
\hline Alho120g em álcool /100kg de PV & 21 & 14 & 48 & 18 & 8 & 60 & 9 & 8 & 13 & 0 \\
\hline Alho $60 \mathrm{~g}$ em água $/ 100 \mathrm{~kg}$ de PV & 48 & 20 & 2 & 64 & 21 & 8 & 12 & 8 & 18 & 0 \\
\hline Alho $120 \mathrm{~g}$ em água $/ 100 \mathrm{~kg}$ de PV & 36 & 6 & 28 & 74 & 26 & 12 & 7 & 2 & 3 & 6 \\
\hline Albendazole ${ }^{1}$ & 9 & 0 & 69 & 0 & 10 & 0 & 11 & 0 & 0 & 0 \\
\hline \multicolumn{11}{|l|}{$2^{\mathrm{a}}$ Administração } \\
\hline Alho $60 \mathrm{~g}$ em álcool $/ 100 \mathrm{~kg}$ de PV & 11 & 0 & 73 & 53 & 6 & 5 & 9 & 0 & 0 & 42 \\
\hline Alho120g em álcool /100kg de PV & 11 & 6 & 9 & 12 & 40 & 26 & 15 & 56 & 0 & 0 \\
\hline Alho $60 \mathrm{~g}$ em água $/ 100 \mathrm{~kg}$ de PV & 15 & 2 & 7 & 34 & 45 & 10 & 19 & 54 & 0 & 0 \\
\hline Alho $120 \mathrm{~g}$ em água $/ 100 \mathrm{~kg}$ de PV & 40 & 0 & 7 & 0 & 20 & 0 & 33 & 0 & 0 & 0 \\
\hline Albendazole ${ }^{1}$ & 0 & 0 & 0 & 0 & 0 & 0 & 0 & 0 & 0 & 0 \\
\hline \multicolumn{11}{|l|}{$3^{\mathrm{a}}$ Administração } \\
\hline Alho $60 \mathrm{~g}$ em álcool $/ 100 \mathrm{~kg}$ de PV & 59 & 86 & 0 & 6 & 9 & 0 & 33 & 8 & 0 & 0 \\
\hline Alho120g em álcool /100kg de PV & 55 & 96 & 6 & 4 & 5 & 0 & 35 & 0 & 0 & 0 \\
\hline Alho $60 \mathrm{~g}$ em água $/ 100 \mathrm{~kg}$ de PV & 28 & 42 & 28 & 10 & 28 & 2 & 28 & 46 & 28 & 0 \\
\hline Alho $120 \mathrm{~g}$ em água $/ 100 \mathrm{~kg}$ de PV & 48 & 80 & 0 & 20 & 9 & 0 & 43 & 0 & 0 & 0 \\
\hline Albendazole ${ }^{1}$ & 0 & 0 & 0 & 0 & 0 & 0 & 0 & 0 & 0 & 0 \\
\hline \multicolumn{11}{|l|}{$4^{\mathrm{a}}$ Administração } \\
\hline Alho $60 \mathrm{~g} \mathrm{em}$ álcool $/ 100 \mathrm{~kg}$ de PV & 83 & 20 & 6 & 4 & 1 & 44 & 10 & 32 & 0 & 0 \\
\hline Alho120g em álcool /100kg de PV & 89 & 52 & 2 & 2 & 1 & 10 & 9 & 36 & 0 & 0 \\
\hline Alho $60 \mathrm{~g}$ em água $/ 100 \mathrm{~kg}$ de PV & 56 & 54 & 11 & 22 & 1 & 2 & 35 & 22 & 0 & 0 \\
\hline Alho $120 \mathrm{~g}$ em água $/ 100 \mathrm{~kg}$ de PV & 82 & 50 & 8 & 2 & 1 & 10 & 9 & 38 & 0 & 0 \\
\hline Albendazole $^{1}$ & 36 & 6 & 28 & 74 & 26 & 12 & 7 & 2 & 3 & 6 \\
\hline \multicolumn{11}{|l|}{ Média das Administrações } \\
\hline Alho $60 \mathrm{~g} \mathrm{em}$ álcool $/ 100 \mathrm{~kg}$ de PV & 41 & 29 & 38 & 31 & 6 & 14 & 15 & 14 & 0 & 13 \\
\hline Alho120g em álcool /100kg de PV & 44 & 42 & 16 & 9 & 14 & 24 & 17 & 25 & 3 & 0 \\
\hline Alho $60 \mathrm{~g}$ em água $/ 100 \mathrm{~kg}$ de PV & 37 & 30 & 12 & 33 & 24 & 6 & 24 & 33 & 12 & 0 \\
\hline Alho $120 \mathrm{~g}$ em água $/ 100 \mathrm{~kg}$ de PV & 52 & 34 & 11 & 24 & 14 & 6 & 23 & 10 & 1 & 2 \\
\hline Albendazole ${ }^{1}$ & 11 & 2 & 24 & 19 & 9 & 3 & 5 & 1 & 1 & 2 \\
\hline
\end{tabular}

Amostras coletadas ao pré e pós-tratamento (7 dia); PV = Peso Vivo. Cooperia spp. (Coop.), Haemonchus spp. (Haem.), Trichostrongylus spp. (Trich.), Ostertagia spp. (Ostert.), Oesophagostomum spp. (Oesoph.). 
populações de helmintos em diferentes épocas do ano (Pimentel Neto e Fonseca, 2002; Ramos et al., 2004), em que o trabalho foi desenvolvido (outono, inverno e primavera). Considerandose o valor médio de todos os animais ao prétratamento, a participação dos gêneros foi de $21 \%$ (Cooperia spp), 38\% (Haemonchus spp.), 15\% (Trichostrongylus spp.), 8\% (Ostertagia spp.) e 18\% (Oesophagostomum spp.). Esses valores guardam relação com àqueles obtidos por Cezar et al. (2010) em rebanho bovino (São Pedro do Sul, RS), ao contatarem predominância dos gêneros Cooperia spp. e Haemonchus spp.

Considerando-se a primeira administração dos produtos, observa-se que não houve crescimento de larvas nas fezes oriundas dos animais tratados com albendazol até o 42。 dia. Após esse período, destaca-se a reinfecção com predominância do gênero Haemonchus spp., Trichostrongylus spp. e Cooperia spp. Para os grupos tratados com alho, os resultados são irregulares; apenas no gênero Cooperia spp. verificou-se diminuição do número de larvas em todos os grupos; para os gêneros Oesophagostomum spp. e Trichostrongylus spp. verificou-se efeito parcial em alguns grupos.

$\mathrm{Na}$ segunda administração, verifica-se maior influência do tratamento com $120 \mathrm{~g}$ de alho sob extração aquosa com indicativo de controle em todos os gêneros. Nos demais grupos de fitoterápicos também se observam influências sobre o crescimento de larvas. Na terceira administração destaca-se o aumento de larvas do gênero Cooperia spp., e, em média, diminuição da população dos demais gêneros. Na quarta administração, a participação dos gêneros Cooperia spp. e Ostertagia spp. manteve-se elevada; para Haemonchus spp. e Trichostrongylus spp. foi baixa e para Oesophagostomum spp. manteve-se o controle.

Para os valores médios dos quatro períodos, houve dificuldade em se apontar influência das diferentes soluções com alho. No entanto, comparando-se os valores de pré com os de póstratamento (quarta aplicação), houve mudança substancial na representatividade da população de larvas infectantes com aumento dos gêneros Cooperia spp. e Ostertagia spp. Para o gênero Oesophagostomum spp. houve controle em todos os grupos tratados com fitoterápico; Para o gênero Trichostrongylus spp., verificou-se menos recuperação de larvas nos grupos tratados com extrato aquoso; Com relação ao Haemonchus spp., houve diminuição substancial na recuperação de larvas em três dos quatro grupos tratados com alho. Esse resultado é importante, pois este gênero é responsável pelas espoliações mais severas e em casos hiperagudos pode ocorrer morte súbita por gastrite hemorrágica (Taylor et al., 2007).
Considerando a eficácia média no período experimental, em todos os grupos com fitoterápico, verificou-se controle parcial dos distintos gêneros de helmintos, havendo melhor resultado com o nível de $120 \mathrm{~g}$ de alho sob extração aquosa.

\section{CONCLUSÕES}

Os resultados demonstram que as diferentes soluções de alho apresentam controle parcial de nematódeos gastrintestinais. As soluções obtidas por extração aquosa são mais adequadas, devido à facilidade de se administrar, pela menor rejeição dos animais ao produto e pelos resultados obtidos. Em todos os grupos tratados com fitoterápico, verificou-se controle parcial dos distintos gêneros de helmintos.

\section{REFERÊNCIA}

AMARAL, F.M.M. et al. Plants and chemical constituents with giardicidal activity. Revista Brasileira de Farmacognosia v.16, supl.1, p.696-720, 2006.

AVANCINI, C.A.M. Sanidade animal na agroecologia: atitudes ecológicas de sanidade animal e plantas medicinais em Medicina Veterinária. Porto Alegre: Fundação Gaia, 1994. 46p.

BATATINHA, M.J.M et al. Efeitos do suco de alho (Allium sativum L.) sobre nematódeos gastrintestinais de caprinos. Ciência Rural, v.34, n.4, p.1265-1266, 2004.

BIANCHIN, I. et al. Eficiência do pó de alho (Allium sativum L.) no controle dos parasitas de bovinos. Campo Grande: Embrapa Gado de Corte, 1999. 31p. (Boletim técnico, 8).

BIANCHIN, I.; CATTO, J.B. Alho desidratado (Allium sativum $\mathrm{L}$.) no controle de nematódeos gastrintestinais em bovinos naturalmente infectados. Ciência Rural, v.34, n.4, p.1267-70, 2004.

BORGES, F.A. et al. Ação anti-ixodídica de uma formulação de ação prolongada (ivermectina+abamectina) contra Boophilus microplus parasitando bovinos naturalmente infestados. A Hora Veterinária. ed. Extra, n.5, p.12-5, 2003

CABARET,J. Animal health problems in organic farming: subjective and objective assessments and farmers actions. Livestock Production Science. v.80, p.99108, 2003.

CARDOSO, C.P. et al. Gastrointestinal parasites in goats. Revista Brasileira de Parasitologia Veterinária, v.21, n.2, p.148-50, 2012.

CATTO, J.B. et al. Sistema de pastejo, rotenona e controle de parasitas em bovinos cruzados: efeito no ganho de peso e no parasitismo. Revista Brasileira de Parasitologia Veterinária, v.18, n.4, p.37-43, 2009.

CEZAR, A.S. et al. Ação anti-helmíntica de diferentes formulações de lactonas macrocíclicas em cepas resistentes de nematódeos de bovinos. Pesquisa Veterinária Brasileira, v.30, n.7, p.523-8, 2010.

CHAGAS, A.C.S. et al. Sensibilidade do carrapato Boophilus microplus a solventes. Ciência Rural, v.33, 
n.1, p.109-14, 2003.

CONDI G.K. et al. Moxidextin-resistant nematodes in cattle in Brazil, Veterinary Parasitology, v.161, n.3/4, p. 213-7, 2009

COSTA, A.J. et al. Avaliação comparativa da ação antihelmíntica e do desenvolvimento ponderal de bezerros tratados com diferentes avermectinas de longa ação. A Hora Veterinária, v.24, n.139, p.31-4, 2004.

FERNANDES, R.M. et al. Ausência da atividade anti-helmíntica de plantas em frangos de corte naturalmente infectados com Heterakis gallinarum. Ciência Rural, v.34, n.5, p.1629-32, 2004.

FORBES, A.B. et al. Sub-clinical parasitism in springborn, beef suckler calves: epidemiology and impact on growth performance during the first grazing season. Veterinary Parasitology, v.104, p.339-44, 2002.

FUJISAWA, H. et al. Biological and chemical stability of garlic-derived allicin. Journal of Agricultural and Food Chemistry, v.56, n.11, p.4229-35, 2008.

FURLONG, J. et al. Controle estratégico do carrapato dos bovinos. A Hora Veterinária, v.23, n.137, p.536, 2004.

GRISI, L. et al. Impacto econômico das principais ectoparasitoses em bovinos no Brasil. A Hora Veterinária, v.21, n.125, p.8-10, 2002.

GORDON, H. McL; WHITLOCK, A.V. A new Technique for counting nematode eggs in sheep faces. Jounal Council Scientific Industrv Research Australia, v.12, p.50-2, 1939.

PIMENTEL NETO, M.; FONSECA, A.H. Epidemiologia das helmintoses pulmonares e gastrintestinais de bezerros em região de baixada do Estado do Rio de
Janeiro. Pesquisa Veterinária Brasileira, v.22, n.4, p.148-52, 2002.

RAMOS, C. I. et al. Epidemiologia das helmintoses gastrintestinais de ovinos no Planalto Catarinense. Ciência Rural, v.34, n.6, p.1889-95, 2004.

ROBERTS, F.H.S.; O'SULLIVAN, J.P. Methods for egges counts and larval cultures for strongyles infesting the gastrointestinal tract of cattle. Autralian Jounal of agricultura Research, v. 1, p.99-102, 1950.

ROEL, A.R. Utilização de plantas com propriedades inseticidas: uma contribuição para o Desenvolvimento Rural Sustentável. Revista Internacional de Desenvolvimento Local, v.1, n.2, p.43-50, 2002.

SANTOS, J.O. et al. A evolução da agricultura orgânica. Revista Brasileira de Gestão Ambiental. v.6, n.1, p.35-41, 2012.

SEQUEIRA, T.C.G.O. Parasitologia animal: animais de produção. EPUB: Rio de Janeiro, 2001. 158p.

TAYLOR,M.A. et al. Veterinary parasitology. Oxford: Blackwell Publishing, 2007. 874p.

UENO, H.; GONÇALVES, V.C. Manual para diagnóstico das helmintoses de ruminantes. Tóquio: Japan International Cooperation Agency, 1998.143p.

VENDRAMIM, J.D.; CASTIGLIONI, E. Aleloquímicos, resistência de plantas e plantas inseticidas. In: GUEDES, J.C.; COSTA, I.D.; CASTIGLIONI, E. (Org.) Bases e Técnicas do Manejo de Insetos. Santa Maria: Pallotti, 2000. p.113-28.

VIEIRA, L.S.; CAVALCANTE, A. C. R. Resistência antihelmíntica em rebanhos caprinos no Estado do Ceará. Pesquisa Veterinária Brasileira, v.9, n.3, p.99-103, 1999. 